PROCEEDINGS OF THE AMERICAN MATHEMATICAL SOCIETY

Volume 124, Number 2, February 1996

\title{
BOUNDS FOR THE OPERATOR NORMS OF SOME NÖRLUND MATRICES
}

\author{
P. D. JOHNSON JR., R. N. MOHAPATRA, AND DAVID ROSS
}

(Communicated by J. Marshall Ash)

\begin{abstract}
Suppose $\left(p_{n}\right)_{n \geq 0}$ is a non-increasing sequence of non-negative numbers with $p_{0}=1, P_{n}=\sum_{j=0}^{n} p_{j}, n=0,1 \ldots$, and $A=A\left(p_{n}\right)=\left(a_{n k}\right)$ is the lower triangular matrix defined by $a_{n k}=p_{n-k} / P_{n}, 0 \leq k \leq n$, and $a_{n k}=0, n<k$. We show that the operator norm of $A$ as a linear operator on $\ell_{p}$ is no greater than $p /(p-1)$, for $1<p<\infty$; this generalizes, yet again, Hardy's inequality for sequences, and simplifies and improves, in this special case, more generally applicable results of D. Borwein, Cass, and Kratz.

When the $p_{n}$ tend to a positive limit, the operator norm of $A$ on $\ell_{p}$ is exactly $p /(p-1)$. We also give some cases when the operator norm of $A$ on $\ell_{p}$ is less than $p /(p-1)$.
\end{abstract}

\section{Introduction}

Hardy's inequality for sequences [8, Theorem 326] asserts that for non-negative numbers $a_{0}, a_{1}, \ldots$ and $1<p<\infty$,

$$
\sum_{n=0}^{\infty} \frac{1}{(n+1)^{p}}\left(\sum_{k=0}^{n} a_{k}\right)^{p} \leq\left(\frac{p}{p-1}\right)^{p} \sum_{k=0}^{\infty} a_{k}^{p},
$$

and that $\left(\frac{p}{p-1}\right)^{p}$ cannot be replaced here by any smaller number. Another way of saying the same thing is to say that the Cesaro matrix

$$
C=\left(c_{n k}\right)_{n, k \geq 0}
$$

defined by $c_{n k}=(n+1)^{-1}, 0 \leq k \leq n$, and $c_{n k}=0, k>n$, determines a continuous linear map

$$
x=\left(x_{k}\right) \rightarrow C x=\left(\sum_{k} c_{n k} x_{k}\right)_{n}=\left(\frac{1}{n+1} \sum_{k=0}^{n} x_{k}\right)_{n}
$$

from $\ell_{p}$ into $\ell_{p}, 1<p<\infty$, with operator norm $\frac{p}{p-1}$.

One area of inquiry inspired by Hardy's inequality focuses on the Nörlund matrices. To obtain one of these, take a sequence $\left(p_{n}\right)_{n \geq 0}$ of non-negative numbers, with $p_{0}>0$, set $P_{n}=\sum_{k=0}^{n} p_{k}, n \geq 0$, and define $A=\bar{A}\left(p_{n}\right)=\left(a_{n k}\right)$ by $a_{n k}=p_{n-k} / P_{n}$, $0 \leq k \leq n$, and $a_{n k}=0, k>n$. Notice that $A\left(p_{n}\right)=A\left(c p_{n}\right)$ for any $c>0$, so

Received by the editors February 4, 1994 and, in revised form, September 7, 1994.

1991 Mathematics Subject Classification. Primary 40G05.

(C)1996 American Mathematical Society 
we may as well assume that $p_{0}=1$. When all the $p_{n}$ are $1, A\left(p_{n}\right)$ is the Cesaro matrix.

It is known (see [1]) that $A\left(p_{n}\right)$ defines a continuous linear map from $\ell_{p}$ to $\ell_{p}$, $1<p<\infty$, when $\left(p_{n}\right)$ is bounded, and quite a lot is known about the operator norm of $A\left(p_{n}\right)$; see [1], [2], [3], and [4]. (Incidentally, $A\left(p_{n}\right)$ always defines a continuous linear map from $\ell_{\infty}$ to $\ell_{\infty}$ with operator norm 1 , because the entries of $A$ are non-negative and its row sums are all 1.) These results, of D. Borwein, F. P. Cass, A. Jakimovski and W. Kratz, are a bit complicated, so we omit their statements. They are usually superior to the results we will give, in that they apply in greater generality, with few restrictions on $\left(p_{n}\right)$. The upper estimate in our Theorem 1 is slightly better than their more general upper estimates, and is a great deal simpler. We do not know if our lower estimate is always, sometimes, or never better than that in [4], but it is much simpler. Our Theorem 2 is just a special case of Theorem 2 in [3], and our proof is no different. The purpose of Theorem 2 in this paper is to provide an alternative result with which to compare Theorem 1.

\section{REsults}

Theorem 1. Suppose that $\left(p_{n}\right)_{n \geq 0}$ is a non-negative, non-increasing sequence of numbers, with $p_{0}=1$. Then the operator norm of $A\left(p_{n}\right)$ on $\ell_{p}$ is no greater than $p /(p-1)$, and no less than $\max (1, \alpha p /(p-1))$, where $\alpha=\liminf _{n \rightarrow \infty} n p_{n} / P_{n}$, for $1<p<\infty$.

Corollary. If the $p_{n}$ of Theorem 1 tend to a positive limit, then the operator norm of $A\left(p_{n}\right)$ is $p /(p-1), 1<p<\infty$.

Theorem 2. Suppose $p_{0}=1, p_{1}, \ldots$ are non-negative. The operator norm of $A\left(p_{n}\right)$ on $\ell_{p}$ is no greater than $\left(\sum_{n=0}^{\infty} p_{n} / P_{n}\right)^{1 / p}$, for $1 \leq p<\infty$.

\section{Proofs}

We shall need a version of a lemma of Davies and Petersen [5], due to Németh $[9]$.

Lemma 1 ([9, Lemma 4]). For $q>1, z_{k} \geq 0$, and $k \leq N$,

$$
\left(\sum_{n=k}^{N} z_{n}\right)^{q} \leq q \sum_{n=k}^{N} z_{n}\left(\sum_{j=n}^{N} z_{j}\right)^{q-1} .
$$

(Here, $N$ may be $\infty$.)

Also, we need a well-known result used implicitly in [8] in the proof of Hardy's inequality. We omit the proof.

Lemma 2. If $\sum a_{n}$ and $\sum b_{n}$ are series with positive terms, $\sum a_{n}$ is divergent, and $b_{n} / a_{n} \rightarrow 1$ as $n \rightarrow \infty$, then $\sum^{N} b_{n} / \sum^{N} a_{n} \rightarrow 1$ as $N \rightarrow \infty$.

Proof of Theorem 1. Suppose $1<p<\infty$. Let $A=A\left(p_{n}\right)$ and $q=p /(p-1)$. We shall prove that the operator norm of $A$ on $\ell_{p}$ is no greater than $q$ by showing that the operator norm of $A^{T}$ on $\ell_{q}$ is no greater than $q$. Suppose $x=\left(x_{n}\right) \in \ell_{q}$ is a 
non-negative sequence. Using conventional notation,

$$
\begin{aligned}
& \left\|A^{T} x\right\|_{q}^{q}=\sum_{k=0}^{\infty}\left(\sum_{n=k}^{\infty} \frac{p_{n-k}}{P_{n}} x_{n}\right)^{q} \\
& \leq q \sum_{k=0}^{\infty} \sum_{n=k}^{\infty} \frac{p_{n-k}}{P_{n}} x_{n}\left(\sum_{j=n}^{\infty} \frac{p_{j-k}}{P_{j}} x_{j}\right)^{q-1} \quad \text { [by Lemma 1] } \\
& \leq q \sum_{k=0}^{\infty} \sum_{n=k}^{\infty} \frac{p_{n-k}}{P_{n}} x_{n}\left(\sum_{j=n}^{\infty} \frac{p_{j-n}}{P_{j}} x_{j}\right)^{q-1} \quad \text { [since the } p_{j} \text { are non-increasing] } \\
& \quad=q \sum_{n=0}^{\infty}\left(\frac{1}{P_{n}} \sum_{k=0}^{n} p_{n-k}\right) x_{n}\left(\sum_{j=n}^{\infty} \frac{p_{j-n}}{P_{j}} x_{j}\right)^{q-1}=q \sum_{n=0}^{\infty}\left(\sum_{j=n}^{\infty} \frac{p_{j-n}}{P_{j}} x_{j}\right)^{q-1} x_{n} \\
& \leq q\left(\sum_{n=0}^{\infty}\left(\sum_{j=n}^{\infty} \frac{p_{j-n}}{P_{j}} x_{j}\right)^{q}\right)^{\frac{1}{p}}\left(\sum_{n=0}^{\infty} x_{n}^{q}\right)^{\frac{1}{q}}=q\left\|A^{T} x\right\|_{q}^{\frac{q}{p}}\|x\|_{q} .
\end{aligned}
$$

From this, $\left\|A^{T} x\right\|_{q} \leq q\|x\|_{q}$ follows as usual.

The proof of the lower estimate of the operator norm of $A$ will be a modification of the proof in [8] that $p /(p-1)$ is the best possible constant in Hardy's inequality. Fix $\delta \in(0,1)$, and suppose $N \geq 1$ is sufficiently large so that $\frac{p_{n}}{P_{n}} \geq \frac{1-\delta}{n} \alpha$ for all $n \geq N$. Then $\frac{p_{n-k}}{P_{n}} \geq \frac{1-\delta}{n} \alpha$ for $n \geq N$ and $0 \leq k \leq n$, because the $p_{j}$ are non-increasing. Suppose $M>N$ and define $x=\left(x_{n}\right)$ by $x_{n}=n^{-1 / p}, N \leq n \leq M$, and $x_{n}=0$ for other values of $n$. We have that

$$
\begin{aligned}
\|A x\|_{p}^{p} & \geq \sum_{n=N}^{M}\left(\frac{1}{P_{n}} \sum_{k=N}^{n} p_{n-k} x_{k}\right)^{p} \\
& \geq \alpha^{p}(1-\delta)^{p} \sum_{n=N}^{M}\left(\frac{1}{n} \sum_{k=N}^{n} k^{-1 / p}\right)^{p} \\
& \geq \alpha^{p}(1-\delta)^{p} \sum_{n=N}^{M}\left(\frac{1}{n} \int_{N}^{n} x^{-1 / p} d x\right)^{p} \\
& =\left(\frac{p}{p-1}\right)^{p} \alpha^{p}(1-\delta)^{p} \sum_{n=N}^{M} \frac{1}{n^{p}}\left(n^{1-1 / p}-N^{1-1 / p}\right)^{p} \\
& =\left(\frac{p}{p-1}\right)^{p} \alpha^{p}(1-\delta)^{p} \rho_{M} \sum_{n=N}^{M} 1 / n \\
& =\left(\frac{p}{p-1}\right)^{p} \alpha^{p}(1-\delta)^{p} \rho_{M}\|x\|_{p}^{p}
\end{aligned}
$$

where $\rho_{M} \rightarrow 1$ as $M \rightarrow \infty$, by Lemma 2 . 
It follows that the operator norm of $A$ is no less than $(1-\delta) \alpha p /(p-1)$, and since $\delta$ was arbitrary, the operator norm of $A$ is no less than $\alpha p /(p-1)$. Since the first column of $A$, the image of the unit coordinate sequence $e_{0}=(1,0,0, \ldots)$, has $\ell_{p}$ norm $\geq 1$, it follows that the operator norm of $A$ is also no less than 1 .

Proof of the corollary. It is easy to see that if $\left(p_{n}\right)$ tends to a positive limit, then $n p_{n} / P_{n} \rightarrow 1$ as $n \rightarrow \infty$.

Proof of Theorem 2. As mentioned above, Theorem 2 here is just a special case of Theorem 2 of [3] (take the $b_{n}$ there to be all 1); we present the straightforward proof, adapted from [3], for the reader's convenience.

Suppose $x=\left(x_{n}\right) \in \ell_{p}$ and $x_{n} \geq 0, n=0,1,2, \ldots$ Let $q=p /(p-1)$. We have

$$
\begin{aligned}
\|A x\|_{p}^{p} & =\sum_{n=0}^{\infty}\left(\sum_{k=0}^{n} \frac{p_{n-k}}{P_{n}} x_{k}\right)^{p} \\
& \leq \sum_{n=0}^{\infty}\left[\left(\sum_{k=0}^{n} \frac{p_{n-k}}{P_{n}}\right)^{1 / q}\left(\sum_{k=0}^{n} \frac{p_{n-k}}{P_{n}} x_{k}^{p}\right)^{1 / p}\right]^{p} \\
& \left.=\sum_{n=0}^{\infty} \sum_{k=0}^{n} \frac{p_{n-k}}{P_{n}} x_{k}^{p} \quad \text { [since } \sum_{k=0}^{n} \frac{p_{n-k}}{P_{n}}=1\right] \\
& =\sum_{k=0}^{\infty}\left(\sum_{n=k}^{\infty} \frac{p_{n-k}}{P_{n}}\right) x_{k}^{p}=\sum_{k=0}^{\infty}\left(\sum_{n=0}^{\infty} \frac{p_{n}}{P_{n+k}}\right) x_{k}^{p} \\
& \leq\left(\sum_{n=0}^{\infty} p_{n} / P_{n}\right) \sum_{k=0}^{\infty} x_{k}^{p}=\left(\sum_{n=0}^{\infty} p_{n} / P_{n}\right)\|x\|_{p}^{p} .
\end{aligned}
$$

\section{Comments}

Regarding Theorem 2, it turns out that $\sum p_{n} / P_{n}<\infty$ only if (and, obviously, if) $\sum p_{n}<\infty$; see Theorem 49, p. 38 of [6]. (The referee tells us that this result is originally due to Dini; we did not succeed in tracking down its origin.)

The original purpose of Theorem 2 in this paper was to show that, under the hypothesis of Theorem 1 , the operator norm of $A\left(p_{n}\right)$ may indeed be less than $q=p /(p-1)$. For instance, when $p_{n}=r^{n}$, for some $r \in(0,1)$, we have

$$
\sum p_{n} / P_{n}=(1-r) \sum \frac{r^{n}}{1-r^{n+1}} \leq \sum r^{n}=\frac{1}{1-r}<q^{p}
$$

whenever $r<1-\left(\frac{1}{q}\right)^{p}$.

However, the referee has directed our attention to a whole continuum of decreasing sequences $\left(p_{n}\right)$ for which the operator norm of $A\left(p_{n}\right)$ is strictly between the bounds of Theorem 1, for every $p \in(1, \infty)$. Suppose $0<r<1$ and set $p_{n}=\left(\begin{array}{c}n+r-1 \\ n\end{array}\right)$. As noted in [1], Hardy in [7] has proven that $A\left(p_{n}\right)$ has operator norm $\frac{\Gamma(r+1) \Gamma\left(\frac{1}{q}\right)}{\Gamma\left(r+\frac{1}{q}\right)}=G(r, q)$ on $\ell_{p}$, for $p>1$. It is a salutary exercise, which we omit here, to see that $\max (1, r q)<G(r, q)<q$ for $0<r<1$ and $q>1$, and that $\alpha=\lim n p_{n} / P_{n}=r$ in this case. This establishes that the operator norm of $A\left(p_{n}\right)$ on $\ell_{p}$ is strictly within the bounds of Theorem 1 . 
Borwein shows in [1], Theorem 1, that $G(\alpha, q)$ is a lower bound of the operator norm of $A\left(p_{n}\right)$ on $\ell_{p}$, with $\alpha=\lim n p_{n} / P_{n}$, for $p>1$ and any positive sequence $\left(p_{n}\right)$, not necessarily monotone, provided $\left(n^{c} p_{n}\right)$ is eventually monotonic for each $c \neq 1-\alpha$. By the remarks above, it follows that when $\left(p_{n}\right)$ is non-increasing, $n p_{n} / P_{n} \rightarrow \alpha$, and $\left(n^{c} p_{n}\right)$ is eventually monotonic for all $c \neq 1-\alpha$ (i.e., when we are in the overlap between Borwein's hypotheses and ours), Borwein's lower bound is better than ours, provided $0<\alpha<1$. In particular, this applies when $p_{n}=(n+1)^{-r}, 0<r<1$. When $p_{n}=(n+1)^{-r}, r \geq 1, \alpha=0$ and both lower bounds are 1 , for all $p>1$. It might be instructive to see if the upper bounds here and in [1] can be much improved in the cases $p_{n}=(n+1)^{-r}, r>0$.

As must be clear from previous remarks, we are greatly indebted to the referee for directing our attention to the right places, as well as for suggestions that improved the results of the first version of this paper.

\section{REFERENCES}

1. David Borwein, Nörlund operators on $\ell_{p}$, Canad. Math. Bull. 36 (1993), 8-14.

2. D. Borwein and F. P. Cass, Nörlund matrices as bounded operators on $\ell_{p}$, Arch. Math. 42 (1984), 464-469.

3. D. Borwein and A. Jakimovski, Matrix operators on $\ell_{P}$, Rocky Mountain J. Math. 9 (1979), 463-477.

4. F. P. Cass and W. Kratz, Nörlund and weighted mean matrices as bounded operators on $\ell_{p}$, Rocky Mountain J. Math. 29 (1990), 59-74.

5. G. S. Davies and G. M. Petersen, On an inequality of Hardy's (II), Quart. J. Math. Oxford Ser. (2) 15 (1964), 35-40.

6. Tomlinson Fort, Infinite series, Oxford University Press, London, 1930.

7. G. H. Hardy, An inequality for Hausdorff means, J. London Math. Soc. 18 (1943), 46-50.

8. G. H. Hardy, J. E. Littlewood, and G. Polya, Inequalities, Cambridge University Press, London, 1934.

9. J. Németh, Generalizations of the Hardy-Littlewood inequality, Acta Sci. Math. (Szeged) 32 (1971), 295-299

Department of Discrete and Statistical Sciences, 120 Math Annex, Auburn UniverSity, Alabama 36849-5307

E-mail address: johnspd@mail.auburn.edu

Department of Mathematics, University of Central Florida, Orlando, Florida 32816-6690

Department of Mathematics, Embry Riddle Aeronautical University, Daytona BEACH, FLORIDA 32114 